\title{
Effects of caloric restriction in the secretion and production of intestinal IgA in mice infected with Salmonella typhimurium
}

\author{
R. A. Jarillo Luna ${ }^{1}$, G. Oliver Aguillón ${ }^{1}$, V. Rivera Aguilar ${ }^{2}$, E. Lara Padilla ${ }^{1}$, J. Pacheco Yépez $^{1}$, \\ B. Martínez Carrillo ${ }^{1}$ and R. Campos Rodríguez ${ }^{1}$ \\ ${ }^{1}$ Sección de Postgrado e Investigación, Escuela Superior de Medicina, Instituto Politécnico Nacional, Plan de San Luis y \\ Díaz Mirón, CP 11340, México DF and ${ }^{2}$ Departamento de Microbiología, UBIPRO, FES-Iztacala, UNAM Av. de los \\ Barrios \#1, Tlalnepantla Edo, de México, CP 54090, México
}

The information about the effects of caloric restriction (CR) on the mucosal immune system is sparse. One study showed that CR reduces the production of proinflammatory cytokines and the expression of the polymeric immunoglobulin receptor (pIgR) ${ }^{(1)}$. Another report indicates that a moderate energy restriction does not affect the secretion of $\operatorname{IgA}$, lactoferrin or lysozyme in human milk ${ }^{(2)}$. Therefore, the aim of this study was to determine in mouse small intestine the effect of CR on IgA intestinal, the IgA+ population of plasmatic cells in the lamina propria, and the evolution of Salmonella typhimurium infection (CRS and ALS groups).

Groups of young Balb/c mice were fed ad libitum (AL), while the CR groups were on alternate days fed ad libitum and fasted. At 6 months of age two groups fed ad libitum, and two submitted to CR were inoculated by intragastric via with $S$. typhimurium. The number of bacteria in faeces was determined by cultivation on S-S agar. IgA levels in the small intestine were quantified by ELISA, while the number of $\operatorname{Ig} \mathrm{A}+$ containing cells in the duodenal mucosa was determined by immunohistochemistry.

The elimination of bacteria in faeces was similar in the ALS and CRS groups. CR decreased the levels of total IgA in the intestine. The groups inoculated with $S$. typhimurium showed that the levels of anti-S. typhimurium IgA were significantly elevated in mice submitted to CR (Fig. 1). The quantity of IgA + cells was less in the non-infected CR than AL groups. Contrarily, the CRS mice showed a significant increase of IgA + cells at 7 days compared to the ALS animals. At 14 days, the number of IgA + cells was similar in both groups (Fig. 2). In conclusion, whereas, CR reduced intestinal IgA levels in non-infected animals, probably by reducing the number of IgA producing cells in the lamina propria, in animals infected with S. typhimurium it increased the quantity of IgA+ cells and IgA levels. However, CR is apparently not advantageous for an organism in the resolution of a primary Gram-negative bacterial infection in the gut.



Fig. 1. Effects of $\mathrm{CR}$ on concentration of anti-S. typhimurium. $\operatorname{IgA}(* P<0.001)$.

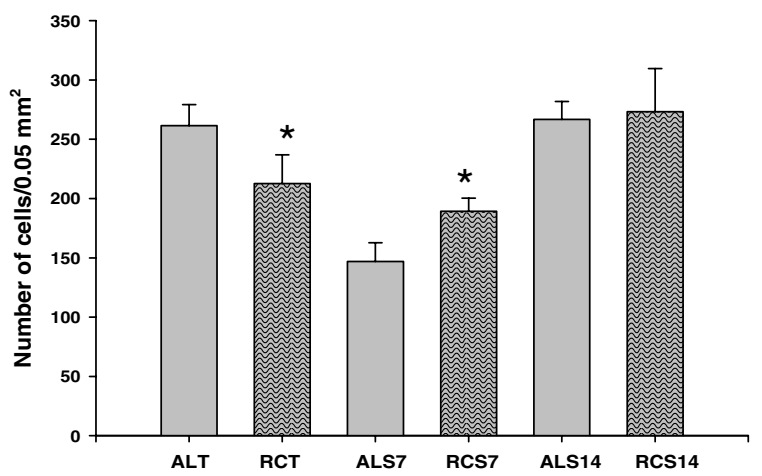

Fig. 2. $\operatorname{Ig} \mathrm{A}+$ cells in lamina propria $(* P<0.05)$.

1. Muthukumar AR, Jolly CA et al. (2000) J Clin Immunol 20, 354-361.

2. Lovelady CA (2004) Adv Exp Med Biol 554, 115-120. 